\title{
Acknowledgement to Reviewers
}

The editors wish to thank the following reviewers for their part in the realization of volume 73, 2011, of ORL.

Adappa Nithin, Philadelphia, Pa. (USA) Adunka Oliver, Chapel Hill, N.C. (USA) Akçam Timur, Ankara (Turkey) Akyildiz Serdar, Izmir (Turkey) Alharbi Fahd, Bad Hersfeld (Germany) Anderson Ilona, Innsbruck (Austria) Araki Koji, Tokorozawa, Saitama (Japan) Bachert Claus, Ghent (Belgium) Bastaninejad Shahin, Tehran (Iran) Basu Devraj, Philadelphia, Pa. (USA) Baumgartner Wolf-Dieter, Wien (Austria) Bayazit Yildirim A., Besevler, Ankara (Turkey) Benazzo Marco, Pavia (Italy) Bhandarkar Naveen, Orange, Calif. (USA) Bian Yansong, Bethesda (USA)

Bianchi Mark S., New Haven, Conn. (USA)

Bigelow Douglas C., Philadelphia, Pa. (USA)

Bing Zhou, Beijing (China)

Blevins Nikolas, Stanford, Calif. (USA)

Bojrab Dennis I., Farmington Hills, Mich. (USA)

Brook Itzhak, Washington, D.C. (USA)

Buchman Craig A., Chapel Hill, N.C. (USA)

Cabra Jesús, Alcázar de San Juan (Spain)

Calabrese Luca, Milan (Italy)

Calzada Gabriel, Downey, Calif. (USA)

Castellanos Paul F., Birmingham, Ala. (USA)

Cernea Claudio R., São Paulo (Brazil)

Chabbert Christian, Montpellier (France)

Chi Fang-Lu, Shanghai (China)

Quan Zheng Chun, Shanghai (China)

Coelho Claudia Barros, Iowa City, Iowa (USA)

Colletti Vittorio, Verona (Italy)

Cremers Christophe, Nijmegen (The Netherlands)

Dagli Muharrem, Ankara (Turkey)

Dallan Iacopo, Lucca (Italy)

De Carlucci Dorival, São Paulo (Brazil)

De Stefano Alessandro, Chieti (Italy)

Desloovere Christian, Leuven (Belgium)
Eisen Marc Daniel, Hartford, Conn. (USA)

Elden Lisa M., Philadelphia, Pa. (USA)

Eleftherios Ferekidis, Athens (Greece)

El Din Mohamed El-Hennawi Diaa, Ismalia (Egypt)

Elmorsy Shawky M., Mansoura (Egypt)

Erkan Alper Nabi, Seyhan, Adana (Turkey)

Esser-Leyding Barbara, Hannover (Germany)

Fang Sheen-Yie, Tainan (Taiwan)

Fawzy Tamer, Giza (Egypt)

Filippo Roberto, Roma (Italy)

Fraysse Bernard, Toulouse (France)

Friedman Oren, Philadelphia, Pa. (USA)

García-Berrocal José Ramon, Madrid (Spain)

Gillespie M. Boyd, Charleston, S.C. (USA)

Gleich Lyon L., Cincinnati, Ohio (USA)

Goldenberg David, Hershey, Pa. (USA)

Gomatos Ilias P., Athens (Greece)

Gourin Christine G., Baltimore, Md. (USA)

Gross Menachem, Jerusalem (Israel)

Gunduz Mehmet, Okayama (Japan)

Guyot Jean-Philippe, Genève (Switzerland)

Haigentz Jr Missak, New York City, N.Y. (USA)

Hakuba Nobuhiro, Toon City (Japan)

Hallworth Richard, Omaha, Nebr. (USA)

Halpin Christopher F., Boston, Mass. (USA)

Halum Stacey, Indianapolis, Ind. (USA)

Hamdan Abdul-Latif, Beirut (Lebanon)

Hamzavi Jafar-Sasan, Wien (Austria)

Han Demin, Beijing (China)

Helbig Silke, Frankfurt (Germany)

Hörmann Karl, Mannheim (Germany)

Hornibrook Jeremy, Christchurch (New Zealand)

Hotomi Muneki, Wakayama (Japan)

Hu Bo Hua, Buffalo, N.Y. (USA)

Hüttenbrink K.-B., Köln (Germany)

Ickenstein Guntram W., Aue (Germany)

Iurato Salvatore, Bari (Italy)

Iwasaki Shinichi, Tokyo (Japan)

\section{KARGER}

Fax +41613061234

E-Mail karger@karger.ch

www.karger.com
(C) 2011 S. Karger AG, Basel 
Jiang Rong-San, Taichung (Taiwan)

Jones Nick S., Nottingham (United Kingdom)

Jutel Marek, Wroclaw (Poland)

Kacker Ashutosh, New York City, N.Y. (USA)

Kanzaki Sho, Tokyo (Japan)

Karaman Murat, Istanbul (Turkey)

Karhuketo Tapio S., Tampere Finland

Kawauchi Hideyuki, Izumo City (Japan)

Kern Robert, Chicago, Ill. (USA)

Kitamura Ken, Tokyo (Japan)

Kitamura Morimasa, Kyoto (Japan)

Kleine-Punte Andrea, Antwerp (Belgium)

Knipper Marlies, Tübingen (Germany)

Korkmaz Mevlit, Istanbul (Turkey)

Kós Marie-Izabel, Geneva (Switzerland)

Kucuk Omer, Atlanta, Ga. (USA)

Kulamarva Gautham, Kasaragod (India)

Kuppersmith Ronald B., Birmingham, Ala. (USA)

Labadie Robert F., Nashville, Tenn. (USA)

Lazaris Andreas C., Athens (Greece)

Leahy Kevin P., Philadelphia, Pa. (USA)

Lee John, Toronto, Ont. (Canada)

Lim David J., Los Angeles, Calif. (USA)

Lin Yung-Song, Yung Kan City (Taiwan)

Lin James, New Orleans, La. (USA)

Liu Tao, Boston, Mass. (USA)

Liu Jeffrey, Philadelphia, Pa. (USA)

Liu Jie, Bethesda, Md. (USA)

Lorens Artur, Warsaw (Poland)

Macrae Phoebe, Christchurch (New Zealand)

Madonini Enzo, Basiglio (Italy)

Malekzadeh Sonya, Washington, D.C. (USA)

Malloy Kelly, Philadelphia, Pa. (USA)

Mandel Jeff, Philadelphia, Pa. (USA)

Manolis Evangelos N., Athens (Greece)

Margolis Robert H., Minneapolis, Minn. (USA)

Matsunobu Takeshi, Tokorozawa (Japan)

Maurer Joachim T., Mannheim (Germany)

Merati Albert, Seattle, Wash. (USA)

Minovi Amir, Bochum (Germany)

Mirza Natasha, Philadelphia, Pa. (USA)

Mishiro Yasuo, Hyogo (Japan)

Motta Sergio, Naples (Italy)

Mowry Sarah, Iowa City, Iowa (USA)

Mühler Roland, Magdeburg (Germany)

Müller Marcus, Tübingen (Germany)

Murofushi Toshihisa, Kawasaki (Japan)

Nacci Andrea, Pisa (Italy)

Nadolnik Liliya, Grodno (Belarus)

Naganawa Shinji, Nagoya (Japan)

Nakagawa Takayuki, Kyoto (Japan)

Nakayama Meijin, Sagamihara (Japan)

Neuburg Marcy, Milwaukee, Wisc. (USA)

Nikolopoulos Thomas, Athens (Greece)

Nuyts Sandra, Leuven (Belgium)
Ogawa Kaoru, Tokyo (Japan)

Ogawa Tetsuya, Nagoya (Japan)

Oghan Fatih, Kutahya (Turkey)

Oh Seung-Ha, Seoul (Republic of Korea)

Olasz L., Pécs (Hungary)

O'Leary Stephen, Melbourne, Vic. (Australia)

Olthoff Arno, Göttingen (Germany)

Onerci Metin, Ankara (Turkey)

Ottaviani Fabrizio, Rome (Italy)

Ozbilen Acar Gul, Istanbul (Turkey)

Ozcan Kursat Murat, Ankara (Turkey)

Ozcan Muge, Ankara (Turkey)

Ozdemir Suleyman, Adana (Turkey)

Ozer Enver, Columbus, Ohio (USA)

Pirodda Antonio, Bologna (Italy)

Probst Rudolf, Zürich (Switzerland)

Quaranta Antonio, Bari (Italy)

Quaranta Nicola, Bari (Italy)

Ragab Ahmed, Shibin El-Koom (Egypt)

Rameh Charbel E., Marseille (France)

Runge Christina L., Milwaukee, Wisc. (USA)

Russ Shirley, Los Angeles, Calif. (USA)

Rybak Leonard P., Springfield, Ill. (USA)

Saraniti Carmelo, Palermo (Italy)

Scarano Emanuele, Rome (Italy)

Schmelzer Andreas, Aarau (Switzerland)

Schwentner Ilona, Innsbruck (Austria)

Senn Pascal, Bern (Switzerland)

Sert Huseyin, Ankara (Turkey)

Shakeel Mohammad, Lucknow (India)

Shepherd Robert, Melbourne, Vic. (Australia)

Shiotani Akihiro, Saitama (Japan)

Speciale Riccardo, Palermo (Italy)

Stavroulaki Pelagia, Volos (Greece)

Stjernquist-Desatnik Anna, Lund (Sweden)

Strojan Primož, Ljubljana (Slovenia)

Sudhoff Holger, Bielefeld (Germany)

Sun Wei, Buffalo, N.Y. (USA)

Suzuki Mikio, Okinawa (Japan)

Tateya Ichiro, Kyoto (Japan)

Taylor Rodney J., Baltimore, Md. (USA)

Thaler Erica R., Philadelphia, Pa. (USA)

Titiz Ali, Ankara (Turkey)

Tokumaru Yutaka, Tokyo (Japan)

Tong Michael C.F., Hong Kong (China)

Toyama Ikuo, Shiga (Japan)

Tsukuda Mamoru, Yokohama (Japan)

Ulug Tuncay, Istanbul (Turkey)

Ünal Murat, Mersin (Turkey)

Ural Ahmet, Ankara (Turkey)

Vander Poorten Vincent, Leuven (Belgium)

Vanderveken Olivier M., Edegem-Antwerp (Belgium)

Vermeire Katrien, Gent (Belgium)

Verse Thomas, Hamburg (Germany)

Vogt Klaus, Rendsburg (Germany) 
Wasowicz Wojciech, Lodz (Poland)

Welling Bradley, Columbus, Ohio (USA)

Widen Judith E., Kansas City, Kans. (USA)

Wild Alan, St. Louis, La. (USA)

Wilhoit Christina S., Charleston, Pa. (USA)

Wing YK, Hong Kong (Hong Kong)

Wise Kim, Auckland (New Zealand)

Wolf Jeffrey S., Baltimore, Md. (USA)

Xi Xin, Beijing (China)

$\mathrm{Xu}$ Yu, Wuhan (China)

Yamashita Daisuke, Kobe City (Japan)
Yang Shi-Ming, Beijing (China)

Yin Shankai, Shanghai (China)

Yoshimoto Seiichi, Tokyo (Japan)

Young Yi-Ho, Taipei (Taiwan)

Zeng Yanjun, Beijing (China)

Zenilman Michael, New York, N.Y. (USA)

Zhang Luo, Beijing (China)

Zhang Gehua, Guangzhou, Guangdong (China)

Zhou Bing, Beijing (China)

Zivic Ljubica B., Kragujevac (Serbia) 